\section{Aplicarión de \\ la Ingeniería Kansei con rades neuronales}

\author{
*Gerardo Avendaño P. PhD \\ **Héctor René Álvarez L. PhD.
}

Fecha de recepción: 17 de abril de 2013 Fecha de aprobación: 2 de mayo de 2013 Pag. 7 a 27

* Postdoctorado Penn State University, Manufacturing Engenniering. Doctorado Universidad Politécnica de Valencia, Métodos Estadísticos Avanzados. Especialización en Ingeniería de Producción, Universidad Distrital Francisco José de Caldas. Pregrado en Ingeniería Química, Universidad Nacional de Colombia.

* Consultor Senior Productiva de Colombia Ltda. Doctorado Universidad Politécnica de Cataluña Aplicaciones Técnicas e Informáticas de La Estadis. Maestría Universidad Nacional de Colombia, Magister en Estadística, Especialización Universidad Distrital Francisco José de Caldas, Especializacion en Ingeniería de Producción, Pregrado en Matemáticas, Universidad Nacional de Colombia.

\section{Resumen}

La Ingeniería Kansei, relaciona las emociones que sienten los consumidores con las características y propiedades que poseen los productos a través de la estimación de un modelo matemático, que permite establecer cuáles tienen mayor relación con las emociones, permitiendo al diseñador, incorporarlas con el fin de activar los factores que las intensifican y dar soluciones efectivas de diseño.

Tradicionalmente, la formulación y estimación del modelo matemático, se hace través de un modelo de regresión múltiple (QT1) y análisis factorial; sin embargo, una de las desventajas es que está condicionado a cumplir los supuestos del modelo. En este trabajo, se muestra cómo las redes

neuronales se pueden aplicar en los estudios de Ingeniería Kansei y nos da resultados similares, permitiendo que se puedan utilizar cuando no se cumplen los supuestos estadísticos.

\section{Palabras clave}

Ingeniería Kansei

Red neuronal Modelo de síntesis Diseño emocional 


\section{Abstract}

Kansei Engineering (emotions) relates that consumers feel with features and properties that have the products through the estimation of a mathematical model, which allows for properties which are high relative to the emotions, allowing the designer incorporate these relations to activate factors which enhance the Kansei design and give effective solutions.

Traditionally the development of the mathematical model and estimation is done through a multiple regression model (QT1) and factor analysis, one of the disadvantages of the estimation of this model is that is conditioned to meet the model assumptions. This paper shows how neural networks can be applied in studies of Kansei Engineering and gives similar results, allowing for use when no statistical assumptions are met.

\section{Keywords}

Kansei Engineering

Neural Network

Model Synthesis

Emotional Design 


\section{Introducción}

T a ingeniería Kansei (IK), es Uuna metodología creada en Japón en los años 80 por Mitsuo Nagamachi. Busca desarrollar productos teniendo en cuenta no sólo las necesidades racionales, sino las emociones y sentimientos del consumidor. Nagamachi (1994), la define como "una metodología de desarrollo de productos orientada al usuario, que establece procedimientos para traducir las percepciones, gustos y sensaciones de productos existentes o conceptos, en términos de soluciones y parámetros de diseño concreto".

Kansei, es una palabra japonesa que hace referencia a los sentimientos y emociones que despierta el producto en el usuario y la imagen psicológica con el mismo respecto del producto. Sin embargo, Nagamachi considera que va más allá de las emociones: "Es un sentimiento personal hacia un producto (imagen o estímulo), que al ser percibido aumenta la calidad.
Conjunto de sentimientos y emociones que se perciben de un producto en el con-texto funcional y su apariencia, que no se expresan en forma directa".

La impresión sicológica y emocional del consumidor, se traduce en soluciones de diseño, a través de valoraciones emocionales realizadas mediante escalas de semántica diferencial (Osgood, 1969) y estos resultados, son integrados en un conjunto de propiedades de los productos, empleando métodos estadísticos especiales.

La Ingeniería Kansei es una metodología para el desarrollo sistemático de nuevas soluciones o de innovaciones. Sin embargo, también puede ser usada como una técnica para mejorar productos y conceptos exitentes, fundamentado en el trabajo de la estimación subjetiva de las emociones actuales comunicadas por el producto que se pretende mejorar. 


\section{Metodología de la ingeniería KANSEI}

Tos diferentes modelos utilizados Uen la IK pretenden traducir las expresiones o palabras emocionales comunicadas por los clientes. El resultado de estas relaciones no necesariamente refleja la estructura verdadera Kansei que tiene de un producto. Schütte (2005), propone un proceso de seis pasos con diferentes métodos que se pueden usar en cada etapa para el desarrollo de un estudio de la Ingeniería Kansei. Al final, enlaza el estudio de las emociones del producto con las propiedades del mismo, en desarrollo. La metodología propuesta por Schütte se centra en tres líneas de acción: la descripción semántica, la descripción de las propiedades del producto y la relación entre ellas.

La descripción semántica produce un espacio factorial, que permite caracterizar los estímulos o productos analizados y producir la matriz de valoraciones Kansei, que describe las propiedades del producto. Estas matrices, se unen en la fase de síntesis, indicando cuáles de las propiedades evocan el impacto semántico. Después que se ha medido el impacto, se conduce una prueba de validez, incluyendo varios tipos de análisis. Una vez realizada la validación, se construye un modelo que describe la forma como el espacio semántico y las propiedades están asociadas.

\subsection{Selección del dominio}

El dominio Kansei se puede describir como la idea perfecta detrás de un producto (Schütte et al, 2004). Se incluye la selección de un grupo objetivo, segmento de mercado, se obtienen muestras y se prepara la información comercial necesaria. Es importante anotar que, el dominio Kansei es una estructura mental abstracta, mientras que los productos representativos son tangibles. A partir de la información recogida, se seleccionan las muestras o estímulos que se van a emplear y se definen las propiedades que se van a evaluar. El dominio puede incluir tanto productos existentes, como conceptos y solución de diseños desconocidos. Las acciones que se realizan en este primer paso están dirigidas a contextualizar el estudio de IK y encontrar representacio- 
nes (productos, imágenes, dibujos, muestras, gráficos $3 \mathrm{D}$, etc.) que cubren en lo posible dicho dominio.

\subsection{Generación del espa- cio semántico}

La generación del espacio semántico se realiza en dos pasos:

- Recogida de palabras Kansei: los palabras Kansei describen el dominio del producto (emocional). Son recogidas de las posibles fuentes disponibles, donde las palabras son usadas para describir el dominio.

- Selección de palabras Kansei usando métodos de reducción de datos: generalmente, es considerado ventajosousar el número original de palabras, ya que si se utiliza alguna selección, se puede perder información valiosa; pero a su vez dificulta la valoración, porque los cuestionarios no son tan efectivos al ser muy extensos. Por lo tanto, la calidad de los datos está afectada por el número de palabras Kansei y la extensión del cuestionario. Es así como se sugiere reducir el número de palabras.

\subsection{Generación del espacio de propiedades}

Los métodos para la construcción del espacio de propiedades no están plenamente desarrollados y la investigación sobre este tema es reducida. En la literatura especializada, Schütte (2004) propone un modelo inicial de tres pasos para la generación del espacio de propiedades.

- Colección: en esta fase se recogen las muestras de productos existentes. A partir de estos productos se identifican las propiedades y las diferentes categorías posibles. Adicionalmente, se incluyen los nuevos conceptos creados por los profesionales que hacen parte del estudio. Se define la imagen de marca y se seleccionan las propiedades.

- Selección: cada grupo de propiedades es priorizada. Para el caso de los productos se utilizan los consumidores. Para los conceptos, se utiliza un grupo de expertos. Para las de imagen de marca se utiliza un grupo de dirección y de Marketing.

- Compilación: se seleccionan los ítems de evaluación integrando todas las propiedades priorizadas. Esta selección puede estar condicionada por requerimientos de 
índole estadístico. Finalmente, se seleccionan los productos representativos que formarán junto con sus categorías, el espacio de propiedades que normalmente se expresan en una matriz llamada de propiedades.

Esta matriz se utiliza en la fase de síntesis y se puede obtener de dos formas:

- Cuando los estímulos se construyen (prototipos). En este caso, la matriz de propiedades se obtiene a partir de una matriz de diseño de experimentos, donde se controlan los ítems que serán seleccionados para cada propiedad.

- Cuando los estímulos ya existen. Este es el caso, en el que por el alto coste se recurre a estímulos existentes, que no pueden ser modificados ni manipulados. Por lo tanto, los estímulos utilizados son los que generan la matriz de propiedades. Es posible, que las propiedades no puedan ser analizadas en su totalidad, pues el espacio está condicionado totalmente a los estímulos disponibles. Otro factor importante, es que los estímulos existentes ya tengan algún parámetro de forma o color, que identifique la marca del estímulo, lo que puede ses- gar la valoración del mismo. La matriz de propiedades depende completamente de los estímulos considerados.

\subsection{Síntesis}

La etapa más importante en un estudio Kansei es la de síntesis, que establece y cuantifica las relaciones existentes entre cada una de las propiedades que poseen los estímulos y los Kansei analizados. Se puede establecer el impacto que tiene globalmente todo el espacio de propiedades sobre ella, pero también se puede determinar qué propiedad tiene un efecto significativo sobre cada uno de ellos.

Para la construcción del espacio de propiedades se utilizan matrices de unos y ceros que tengan propiedades de ortogonalidad, como las que poseen las matrices de diseño de experimentos factoriales. Esta es una característica aconsejable para mejorar la fiabilidad de los resultados de la etapa de síntesis y por esta razón, se recomienda tomar como referencia una matriz de diseño de experimentos en la construcción de la de propiedades, o por lo menos que sea muy similar en su estructura. 
Para establecer y cuantificar la Donde $\mathrm{Y}$ es la valoración Kansei relación entre los ítems de las pro- promedio de todos los individuos piedades de los estímulos y la que se incluyen en el estudio y valoración de cada palabra Kansei, $\quad \mathrm{X}_{1}, \mathrm{X}_{2}, \ldots, \mathrm{X}_{\mathrm{p}}$ son las variables que se construye un modelo matemático indican los ítems evaluados de las con la siguiente forma:

$$
Y=f\left(X_{1}, \ldots, X_{p}\right)
$$
(1) ítems lij de la tabla de propiedades). propiedades de los estímulos (cada variable $\mathrm{Xj}$ representa un de los 


\section{Modelo de síntesis: Teoría de Cuantificación de Hayashi (QT1)}

E propiedades y el semántico, donde se estima la contribución de los ítems de cada propiedad, sobre la valoración Kansei realizada por los consumidores. Está basado en el modelo de regresión lineal y es el llamado Teoría de Cuantificación QT1 (Nagamachi, 2008). El método QT1 fue propuesto por Hayashi (1952), permite formular un método de cuantificación de las relaciones existentes entre el conjunto de variables categóricas sobre una numérica.

Para expresar formalmente el modelo de la ecuación (1), se definen las variables indicadoras $X_{\mathrm{jk}}$ (i) que identifican cada ítem de la propiedad en el espacio de propiedades, de la siguiente forma:

$$
X_{j k}(i)=\left\{\begin{array}{cc}
1 & \text { Si el } i \text {-édimo estímulo poseeel } \\
0 & \text { item } j \text { en la propiedad } k \text { para } \\
\text { otro caso }
\end{array}\right.
$$

A partir de las variables indicadoras $X_{\mathrm{j} k}(i)$ se deben estimar los parámetros $\beta_{\mathrm{jk}}$ del modelo:

$$
Y_{i}=\sum_{j} \sum_{k} \beta_{j k} X_{j k}(i)+\varepsilon_{j k}
$$

Hayashillamó a los $\beta_{\mathrm{j} k}$ como los puntajes de las Categorías (Category Scores, CS) y propuso estimarlos maximizando el coeficiente de correlación entre $p$ y $Y_{j}$ y $X_{11}+$ $\ldots+X_{\mathrm{mkm}}$. Existen varias versiones del algoritmo de estimación de los CS; los más importantes son el de Tanaka (1976) que también es basado en la maximización de la correlación p y el de Murai (1988), que minimiza la suma de los errores al cuadrado:

$$
L=\sum_{i}\left(Y_{i}-\hat{Y}_{i}\right)^{2}=\sum_{i}\left(Y_{i}-\sum_{j} \sum_{k} \beta_{j k} X_{j k}(i)\right)^{2}
$$




\subsection{Modelo de síntesis en la práctica}

Cuando se estima el modelo de síntesis a través de la regresión múltiple (que Schütte llama la regresión de Hayashi), en realidad se estiman dos modelos: uno (M1) para las propiedades y otro (M2) para los ítems de cada propiedad. En el modelo M1, se incluyen las variables $\mathrm{X}_{\mathrm{jk}}$ pero como variables Dummy, del modelo de regresión clásico, donde en cada propiedad se incluyen $\mathrm{k}_{\mathrm{j}}-1$ ítems; en el modelo M2, se incluyen todas las variables $\mathrm{X}_{\mathrm{jk}}$. De esta forma con el M1 se hace la estimación del efecto de las propiedades sobre las valoraciones Kansei y con el M2 se hace la estimación del efecto de los ítems de cada propiedad sobre las valoraciones Kansei.

Usando el modelo M1 se puede estimar el coeficiente de determinación $\mathrm{R}^{2}$ y los Coeficientes de Correlación Parcial (CCP) para cada $X_{j k}$. El coeficiente de determinación mide el efecto global del espacio de propiedades sobre cada valoración Kansei y el CCP, mide el efecto de cada propiedad sobre esta. El modelo M2 permite estimar los (CS) Category Scores o 口jk que cuantifica el efecto de cada ítem (de cada propiedad) sobre la valoración Kansei.
Para establecer la significancia del $\mathrm{R}^{2}$ se utiliza el análisis de varianza del modelo de regresión. En caso de resultar significante la contribución global del espacio de propiedades, se establece la significancia del CCP. Aunque Schütte y Eklund (2004) plantean que CCP $>70 \%$ se puede considerar significativo, pero no se hace una justificación técnica de este criterio. También es posible usar una prueba estadística para la significancia del CCP y esta es descrita en Álvarez (2009).

Para los $\beta_{\mathrm{jk}}$ no se hace prueba de significancia, simplemente interesa establecer su signo: si es positivo, genera un efecto de intensificación positiva del Kansei y si es negativo, el efecto es de intensificación negativo. Con este criterio se establecen las conclusiones del estudio así:

En el ejemplo del diseño de un envase cosmético (Álvarez, 2009), se describe como se interpreta. En esta aplicación se analizaron ocho estímulos de un envase para crema. En un ejemplo del diseño Kansei de un envase para un producto cosmético, se analizaron ocho estímulos y se estudiaron cinco propiedades: color, acabado, material, forma y grosor de pared, en este caso se analizó el Kansei Elegante. El efecto global es $\mathrm{R}^{2}=99,9 \%$. 
Para establecer el efecto de cada de los Category Score estimados propiedad, se calcula el CCP y se del modelo QT1, se puede concluir observa que el color, material y groso que los ítems que contribuyen positide pared tienen CCP significativos vamente al Kansei estudiado son: y las propiedades que generan un color, monocolor; material, plástico y efecto significativo son color, material el grosor de pared, delgada. y grosor de pared. Finalmente, a partir

Tabla 1. Resultados del modelo de síntesis para el Kansei "Elegante"

\begin{tabular}{|c|l|l|l|l|l|}
\hline PROPIEDAD & \multicolumn{1}{|c|}{ CCP } & \multicolumn{1}{|c|}{ ITEM } & \multicolumn{2}{|c|}{ CATEGORY SCORE } & CS \\
\hline \multirow{2}{*}{ Color } & $0,99^{*}$ & Monocolor & & & 0,4271 \\
\hline & $\mathrm{P}=0,0000$ & Multicolor & & & $-0,4271$ \\
\hline \multirow{2}{*}{ Acabado } & 0,55 & Brillante & & & $-0,0083$ \\
\hline & $\mathrm{P}=0,1650$ & Mate & & & 0.0083 \\
\hline \multirow{2}{*}{ Material } & $0,99^{*}$ & Plástico & & & 0,2429 \\
\hline & $\mathrm{P}=0,0000$ & Vidrio & & & $-0,2429$ \\
\hline \multirow{2}{*}{ Forma } & 0,10 & Irregular & & & $-0,0013$ \\
\hline & $\mathrm{P}=0.4527$ & Lineal & & & 0,0013 \\
\hline \multirow{2}{*}{$\begin{array}{c}\text { Grosor de } \\
\text { Pared }\end{array}$} & $0,99^{*}$ & Delgado & & & 0,0917 \\
\hline & $\mathrm{P}=0,0005$ & Grueso & & & $-0,0917$ \\
\hline
\end{tabular}

Fuente. Elaboración de los autores. 


\section{Redes neuronales e ingeniería Kansei}

L a aplicación de redes neuronales a los estudios de ingeniería Kansei, se han planteado frente a la restricción que tienen los modelos estadísticos que permiten relacionar el espacio semántico y la matriz de propiedades de los productos que se están analizando.

La primera restricción, es que el supuesto básico de normalidad de las observaciones por lo general, no se cumplen, debido a que la valoración de la emocionalidad, se hace en escala de Osgood y esta se mide en escala discreta y no en continuo; es una limitación que han tenido la gran mayoría de estudios de IK. Una forma de manejar esta limitación es usando muestras grandes, pero es evidente que logísticamente, realizar estudios de Ingeniería Kansei en estas proporciones se hace complicado y costoso (Álvarez, 2009).

El utilizar el modelamiento nacido de la inteligencia artificial y análisis de sistemas de información permite obviar este supuesto, la construcción del modelo no está basada en los supuestos estadísticos.

Otra dificultad que se presenta tiene que ver con lo que plantea el Dr. Nagamachi (2006); algunos Kansei tienen características lineales, pero otros no, es como el caso del Kansei Estilizado que se incrementa linealmente con la propiedad longitud de diseño, pero al evaluar el Kansei Bello, este muestra un comportamiento no lineal al incrementar la longitud (en envases). Si posee esta característica no lineal, no sería correcto aplicar el análisis estadístico basado en regresión lineal.

Nagamachi sugiere que para evitar el efecto de la linealidad y algunos supuestos estadísticos como la normalidad, se puede aplicar otras metodologías como la Rough Sets (conjuntos aproximados) o las redes neuronales. 
El Dr. Ishihara, quien trabaja conjuntamente con el Dr. Nagamachi, fue quien propuso por primera vez aplicar las redes neuronales a la IK con un entorno de sistemas de aprendizaje. Planteó un modelo aplicado al de síntesis (Ishihara et al 1996) y pensó en usar un modelo multicapa como (figura 1.)

Figura 1. Estructura neuronal artificial aplicada a la IK

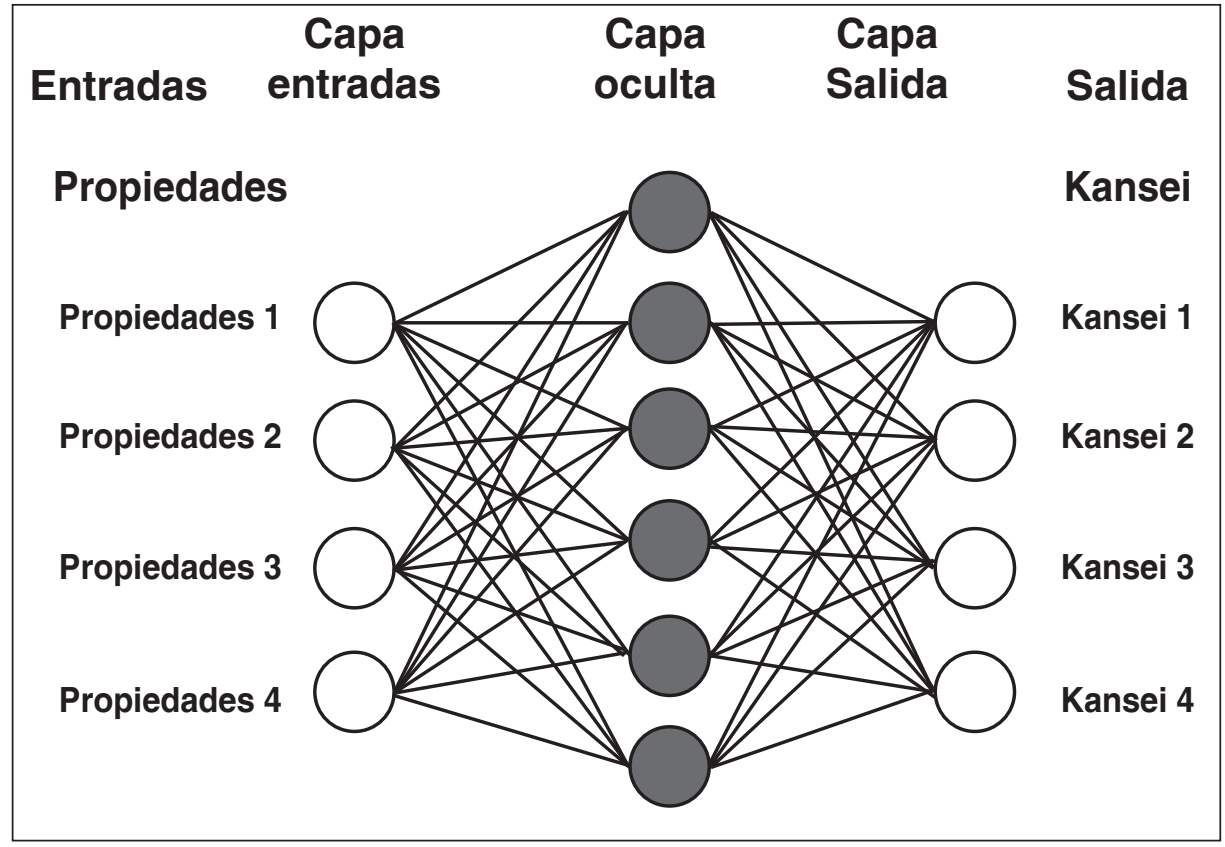

Fuente. Elaborado de los autores.
Guan y Lin, (2001), plantearon aplicar las redes neuronales en el diseño emocional de teléfonos celulares, allí ellos abordaron la problemática que implicaba tener características de productos con muchas categorías, y con un gran número de estímulos.

\section{Capa}

entradas
Capa oculta

\section{Salida}

Kansei

Kansei 1

Kansei 2

Kansei 3

Kansei 4 


\section{Aplicación en diseño de un envase}

\section{cosmético}

Dara describir la aplicación de las 1 redes neuronales en la Ingeniería Kansei se utilizará un ejemplo que muestra Álvarez (2009), del diseño emocional de un envase cosmético. En la industria cosmética uno de los factores determinantes para la aceptación y el éxito de un producto cosmético es el llamado envase cosmético.

Este tipo de productos se asocian a las características holísticas emocionales, las cuales se complementan con las funcionales. El caso de los envases para desodorantes para hombre, es un típico ejemplo donde además de la fragancia y la funcionalidad del envase, son muy importantes los factores emocionales asociados la marca y la visión holística varonil mezclada con la elegancia.

En esta aplicación, se tuvo especial atención en los aspectos emocionales del envase es decir, aquellos que permitan establecer las características finales del producto y su presentación. Se trata del diseño Kansei de un envase de desodorante en espray perfumado para caballero, donde se rediseñó bajo una óptica emocional y desde un claro reposicionamiento del producto.

Desde el punto de vista de la Ingeniería Kansei, este tipo de estudios son los más comunes que se realizan en la industria cosmética, diseño o rediseño de envases, comparándolos con productos Benchmarking, de cara a tratar de identificar qué propiedades del envase se asocian a valores emocionales de.los productos, que.ya están posicionados. Por esta razón, estos estudios deben realizarse con cuidado y detalle, porque muchos ya son conocidos e identificados por la forma o la estética del envase, por lo tanto, se debe ocultar la marca en el momento de exposición para la valoración emocional. 


\subsection{Selección del dominio Kansei}

El objetivo del estudio, consistía en establecer qué ajustes debian hacerse al diseño del envase para lograr que el consumidor pudiera percibir valores emocionales (Kansei), de forma tal que fuera comparado con otros conocidos y de alto valor. El segmento de mercado a quien iba dirigido el estudio, estaba definido por el objetivo del estudio, debido a que se queria posicionar el producto de referencia dentro del estrato medio. Las características emocionales de productos Bench de segmento alto (desodorantes con alto valor de marca), dirigido a hombres mayores de 20 años y de segmento socioeconómico medio. Se tomaron en cuenta productos representativos del mercado de desodorantes perfumados y con prestigio existentes en el mercado cosmético español, por tanto, se incluyeron diseños reales.

\subsection{Selección del dominio Kansei}

En esta fase, se definio la selección de la muestra de consumidores, se seleccionaron los estímulos a evaluar, y la planeación logística para la ejecución. Se tomó como muestra a estudiantes de una universidad los cuales fueron encuestados previamente, con el fin de que cumplieran con los requisitos básicos de mayores de 20 años y usuarios de desodorante en espray. Finalmente participaron 27 estudiantes en el estudio que se consideró como exploratorio; aparte se seleccionaron cinco estudiantes distintos para realizar la prueba piloto con la cual se validó el método de captura de datos y la forma de exposición de los estímulos; a partir de allí se diseño una guía del estudio de campo.

Para la selección de estímulos, se visitaron las tiendas de venta de cosméticos de mayor prestigio asi mismo, se consultaron catálogos y revistas especializadas de cosméticos $y$ se seleccionaron inicialmente 25 productos de alto valor de marca. De estos, se excluyeron aquellos envases cuya forma difería radicalmente del producto de estudio. En esencia, lo que se quería era rediseñar el envase sin hacer cambios radicales de concepto y al final se seleccionaron nueve productos (incluyendo el de rediseño). Es importante señalar que para la selección de los estímulos se utilizaron dos criterios, propiedades ya definidas de los envases de desodorante de espray tales como: la combinación de color y continuidad de la tapaenvase; altura total, textura del material; alcance funcional al 
dispensar el producto y relación tapaenvase. El otro criterio que se utilizó, fue el de garantizar que por lo menos la mitad de los estímulos tuvieron las características anteriormente mencionadas, esto con el propósito de mantener el balance de cada una de las categorías consideradas.

La matriz de propiedades (figura 2), define las variables regresoras del modelo. Es importante notar que la columna continuidad tiene un número de ceros y unos no balanceado, por esto, la matriz pierde la propiedad de ortogonalidad y afecta al modelo y los supuestos.

Para la construcción del espacio se- mántico, se recolectaron palabras Kansei, tomando como fuentes los informes de estudios comerciaes realizados anteriormente por la compañía; además, se consultaron a los clientes directamente en las tiendas, revistas especializadas de cosmética, catálogos y personal de venta. En total se recogieron 65 palabras Kansei, que luego del proceso de compresión a través del diagrama de afinidad y el análisis cluster, se redujeron a diez palabras Kansei, ellas fueron: fresco, elegante, moderno, estilizado, deportivo, protector, exclusivo, estético, alegre y dinámico.

Figura 2. Matriz de propiedades del envase de desodorante

\begin{tabular}{|c|c|c|c|c|c|c|c|c|c|c|c|c|}
\hline & \multicolumn{2}{|c|}{$\begin{array}{l}\text { Color } \\
\text { tapa- } \\
\text { envase }\end{array}$} & \multicolumn{2}{|c|}{$\begin{array}{l}\text { Altura } \\
\text { envase }\end{array}$} & \multicolumn{2}{|c|}{$\begin{array}{l}\text { Continuidad del } \\
\text { envase }\end{array}$} & \multicolumn{2}{|c|}{ Alcance funcional } & \multicolumn{2}{|c|}{$\begin{array}{l}\text { Relación } \\
\text { envasetapa }\end{array}$} & \multicolumn{2}{|c|}{ Textura } \\
\hline & $\begin{array}{l}\text { Un } \\
\text { color }\end{array}$ & dos & Alto & Medio & Continuo & Discuntin & Clase A & Clase B & Alta & Normal & Media & Suave \\
\hline & 0 & 1 & 1 & 0 & 1 & 0 & 0 & 1 & 1 & 0 & 1 & 0 \\
\hline$\sigma^{2}$ & 1 & 0 & 0 & 1 & 0 & 1 & 1 & 0 & 0 & 1 & 0 & 1 \\
\hline & 1 & 0 & 0 & 1 & 0 & 1 & 1 & 0 & 0 & 1 & 1 & 0 \\
\hline & 1 & 0 & 1 & 0 & 0 & 1 & 1 & 0 & 1 & 0 & 0 & 1 \\
\hline & 0 & 1 & 1 & 0 & 1 & 0 & 0 & 1 & 0 & 1 & 1 & 0 \\
\hline & 1 & 0 & 0 & 1 & 0 & 1 & 1 & 0 & 0 & 1 & 1 & 0 \\
\hline 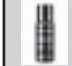 & 0 & 1 & 1 & 0 & 0 & 1 & 0 & 1 & 1 & 0 & 0 & 1 \\
\hline & 1 & 0 & 0 & 1 & 0 & 1 & 0 & 1 & 1 & 0 & 0 & 1 \\
\hline & 0 & 1 & 1 & 0 & 0 & 1 & 0 & 1 & 0 & 1 & 1 & 0 \\
\hline
\end{tabular}

Fuente. Elaboración de los autores. 


\section{Análisis de los resultados}

P ara el análisis e interpretación de los resultados del modelo,se consideró primero, el estudio de posicionamiento emocional de los estímulos de acuerdo a las valoraciones Kansei realizadas por los consumidores, En particular se obtuvo que los estímulos cuatro y seis se observan como los más elegantes y estéticos, mientras que el uno no se muestra como elegante ni estético, pero si alegre y deportivo; el cinco se muestra fresco. Por otro lado, el siete, que se estaba rediseñando, se mostró protector y estilizado, pero poco elegante.

Para el análisis del modelo de síntesis se considero el segundo Kansei que se refiere a Elegante. Este hace parte del valor de marca

con que se ha diseñado el estimulo de referencia. Usando el método estadístico con el QT1 (tabla 2). Se observa que el espacio de propiedades que se está analizando, aporta un $93.3 \%$ a la variabilidad de la valoración del Kansei. Al entrar a analizar en detalle las propiedades, se encuentra que la continuidad del envase, junto con el alcance, son las significativas es decir, que las otras no le aportan realmente al Kansei Elegante. De esta forma, concluye que, la continuidad envase-tapa y el alcance clase $A$, son las que aportan positivamente al Kansei Elegante. Al analizar en detalle el estímulo de referencia ya se habia notado que es discontinuo entre envase-tapa y el alcance es de clase B. 
Tabla 2. Evaluación del modelo de síntesis del Kansei Elegante.

\begin{tabular}{|c|c|c|c|c|c|}
\hline $\mathbf{R} 2$ & $\begin{array}{l}\text { Propieda- } \\
\text { des }\end{array}$ & CCP & Categorias & CS & -1 \\
\hline \multirow{12}{*}{$93.3 \%$} & \multirow{2}{*}{$\begin{array}{l}\text { Color Tapa/ } \\
\text { envase }\end{array}$} & \multirow{2}{*}{0,49} & \multirow{2}{*}{$\begin{array}{l}\text { Uno } \\
\text { Dos }\end{array}$} & $-0,51$ & \\
\hline & & & & 0,51 & \\
\hline & \multirow{2}{*}{ Altura } & \multirow{2}{*}{0,38} & \multirow{2}{*}{$\begin{array}{l}\text { Alto } \\
\text { Medio }\end{array}$} & $-0,49$ & \\
\hline & & & & 0,49 & \\
\hline & \multirow{2}{*}{ Continuidad } & \multirow{2}{*}{0,75} & \multirow{2}{*}{$\begin{array}{l}\text { Continuo } \\
\text { Discontinuo }\end{array}$} & 1,10 & \\
\hline & & & & $-1,10$ & \\
\hline & \multirow{2}{*}{ Alcance } & \multirow{2}{*}{0,67} & \multirow{2}{*}{$\begin{array}{l}\text { Clase A } \\
\text { Clase B }\end{array}$} & 0,89 & \\
\hline & & & & $-0,89$ & \\
\hline & \multirow{2}{*}{$\begin{array}{l}\text { Relación } \\
\text { envase/ } \\
\text { tapa }\end{array}$} & \multirow[b]{2}{*}{0,31} & Alta & $-0,43$ & \\
\hline & & & Normal & 0,43 & \\
\hline & \multirow{2}{*}{ Textura } & \multirow{2}{*}{0,20} & \multirow{2}{*}{$\begin{array}{l}\text { Medio } \\
\text { Suave }\end{array}$} & $-0,65$ & \\
\hline & & & & 0,65 & \\
\hline
\end{tabular}

Fuente. Elaboración de los autores.

Al realizar la validación del modelo de síntesis que lo podemos escribir de la forma:

$Y_{\text {Elegante }=4,7-1,7}$ Color $_{\text {apalenvase }}-1,3$ Altura +2 Continuidad +2 Alcance + 0,1 Relación $n_{\text {Envaseltapa }}-$ 0,5 Textura

Se puede observar que la continui- representa las propiedades, una dad y el alcance son los que tienen capa intermedia de procesamiento coeficientes más altos dentro del y en la capa de salida se represenmodelo, siendo coherente con los tan los Kansei.

parámetros. Al revisar los supuestos, se encuentra que este modelo tiene una alta colinealidad; así mismo, al hacer la prueba de normalidad de los residuos, no cumple.

Se muestra la red neuronal tipo Backpropagation (figura 3) Avendaño (2009), donde la primera capa

Específicamente, para el kansei Elegante $(Y)$ se entrenó una red tipo Backpropagation con los datos encontrados (tabla 3). Se encontró una red óptima de seis nodos en la capa de entrada (que representan las propiedades $\mathrm{X} 1$ a $\mathrm{X} 6$ : color, altura continuidad, alcance, relación 
y textura respectivamente); una que representa el Kansei Elegante capa oculta y una capa de salida (Y) dando un valor $r$ de 0,95.

\section{Tabla 3. Valores para entrenamiento de la red neuronal}

\begin{tabular}{|l|l|l|l|l|l|l|}
\hline $\mathrm{X} 1$ & $\mathrm{X} 2$ & $\mathrm{X} 3$ & $\mathrm{X} 4$ & $\mathrm{X} 5$ & $\mathrm{X} 6$ & $\mathrm{Y} 1$ \\
\hline 0 & 1 & 1 & 0 & 1 & 1 & 4,2 \\
\hline 1 & 0 & 0 & 1 & 0 & 0 & 4,2 \\
\hline 1 & 0 & 0 & 1 & 0 & 1 & 4,3 \\
\hline 1 & 1 & 0 & 1 & 1 & 0 & 5,3 \\
\hline 0 & 1 & 1 & 0 & 0 & 1 & 4,4 \\
\hline 1 & 0 & 0 & 1 & 0 & 1 & 4,8 \\
\hline 0 & 1 & 0 & 0 & 1 & 0 & 5,0 \\
\hline 1 & 0 & 0 & 0 & 1 & 0 & 4,0 \\
\hline 0 & 1 & 0 & 0 & 0 & 1 & 5,2 \\
\hline
\end{tabular}

Fuente. Elaboración de los autores. 


\title{
7. Conclusiones
}

\begin{abstract}
quí se ha demostrado con un A ejemplo, como a través del uso de las redes neuronales, se obtienen resultados similares a los obtenidos con el modelo clásico, a pesar de las falencia encontrados. En este caso, la red neuronal está validando los resultados del modelo, pero en ocasiones esto no ocurre, especialmente cuando la matriz dista demasiado de ser ortogonal. También, se puede mostrar que las redes neuronales son muy útiles

cuando se tienen un mayor número de propiedades (columnas, en la matriz de propiedades), que de estímulos. Esta situación hace que no pueda estimarse el modelo de síntesis con regresión clásica ni QT1 y en tal caso la metodología de redes neuronales es más robusta e independiente de estos supuestos.La red encontrada es óptima ya que da un $r$ de 0.95 superior al valor del modelo matemático planteado.
\end{abstract}




\section{Referencias}

Álvarez H. R. (2006). Proceso de Síntesis en un estudio de Ingeniería Kansei. Disponible en: http://www.ingenieria-kansei.com/papersintesis.pdf

Álvarez H. R. (2009). Análisis de valoraciones atípicas en los estudios de ingeniería Kansei: consideraciones estadísticas y prácticas. Tesis Doctoral. Universidad Politécnica de Cataluña.

Avendaño, G. (2009). Aplicación al control de calidad. Editorial ARFO.

Guan, S. S. y Lin Y.C. (2001). A Study on the Color and Style Collocation of Mobile Phones Using Neural Network Method. Journal of the Chinese Institute of Industrial Engineers, 18(6), 84-94

Hayashi, C. (1952). On the prediction of phenomena from qualitative data on the quantification of qualitative data from the mathematic-statistical point of view. Annals of Mathematical statistics. Vol 3 (69).

Hayashi C. (1974). Methods of Quantification. Tokyo Keizai Shipo Publishing Co. Tokyo.

Ikeda G, Gagai H. y Sagara Y. (2004) Development of Food Kansei Model and Its Application for Designing Tastes and Favors of Green Tea Beverages. Food Sci. Technol. Res. Vol 10 (4) p 396-404

Ishihara, S., Ishihara, K., Nagamachi, M. and Matsubara, Y. (1996). Neural network approach for Kansei analysis on milk carton design. Proceedings of Human Factors in Organizational Design and Management - V, Elsevier Science B.V., Breckenridge, USA, p. 7-12. 
Komazawa, T. y Hayashi, C. (1976). A Statistical Method for Quantification of Categorical Data and its Applications to Medical Science. de Dombal, F. T. y Gremy, F. (ed.), North-Holland Publishing Company

Lin R., Lin C.Y. y Wong J. (1996). An application of multidimensional scaling in product semantics. Int Journal of Industrial Ergonomics 18. Elsevier. pp 193-204

Murai T., Kudo Y and Akama S. (2006). A role of Granularity and Background Knowledge in Reasoning Process. Kansei Engineering International. Vol 6. No 3 p 41-46

Nagamachi, M. (1994). Kansei Engineering: An ergonomic technology for a product development. Proceedings of IEA '94. 1220-122

Nagamachi, M. (2007). Perspectives and new trend of Kansei/Affective Engineering. 1st European Conference on Affective Design and Kansei Engineering. Lund University \& Linköping University

Nagamachi, M, Nishino, T and Okuo, H (2007). An application of kansei Engineering and Rough Set Model to designing a comprehensive ball pen. Proceedings Of the QMOD 2007

Osgood C. E. y Suci G. J. (1969). Factor Analysis of Meaning. Semantic Differential Technique - a Source Book. Osgood, C. E. and Snider J. G. (ed) Aldine Publishing Company, Chicago, p42-55

Schütte S., Ecklund J, Axellson J.R.C., y Nagamachi, M (2004). Concepts, methods and tools in Kansei Engineering" Theoretical Issues in Ergonomics Science, May-June 2004, vol. 5, no. 3, 214-231

Schütte S. (2005). Engineering Emotional Values in Product Design: Kansei Engineering in Development. Thesis PhD. Linköping Studies in Science and Technology. Linköpings Universitet

Tanaka, Y. (1979). Review of the Methods of Quantification. Environmental Health Perspectives. Vol 32 pg: 113-123. 